\title{
Coverillustration
}

\section{Is the brain overrated?}

$\mathrm{P}$ receding the Cambrian explosion by millions of years, jellyfish have a history that goes back at least 650 million years, and they were probably the first multicellular organisms to become motile. Most jellyfish have rather simple visual mechanisms that suggest their humble beginnings, but in vision one class of these creatures stands out.

The cubozoan jellyfish, a class in the phylum Cnidaria, are visually precocious and have surprisingly sophisticated ocular anatomy. While other jellyfish have primitive eyes, the cubozoans have camera style eyes, much like squid or even vertebrates, although distinct differences exist. This class of jellyfish has members that vary in size, and Tripedalia cystophora, seen on this month's cover, is one of the smallest of the jellyfish at 10-12 mm long. In the Caribbean, this species preys upon copepods, a tiny shrimp-like zooplankton, and must rely upon its vision to keep it in the shafts of light that filter between the mangrove roots where the copepods swarm.

The approximately 14 species of cubozoans are cuboidal in shape, as their name suggests, with roughly square sides to the bell. The stinger laden tentacles extend from each of the four corners of the bell. Each corner of the squareshaped bell sports a tentacle or group of tentacles that can contract or extend, often for many metres behind or below the bell. These tentacles contain the nematocysts that contain the poison. One of the family, Chironex fleckeri, contains perhaps the deadliest venom known; capable of killing humans within minutes in a sufficient envenomation.

Jellyfish are gelatinous diploblasts and are radially symmetrical. Their neural network is organised into subumbrellar rings and ganglia, but no central processing centre that we would consider a brain. Evolutionarily, jellyfish are probably descendants of the sponges but represent a cul de sac without further descendants. A different, and now extinct, creature from the Ediacaran fauna may have been more closely related to the creatures of the Cambrian explosion. But, the surprisingly motile and often poisonous jellyfish have much to teach us about ocular anatomy and physiology.

Near the lower edge of the bell, on the exterior surface, there are four flowerlike structures, known as rhopalia, dangling like a watch on a chain. Two rhopalia can be seen on the cover photograph

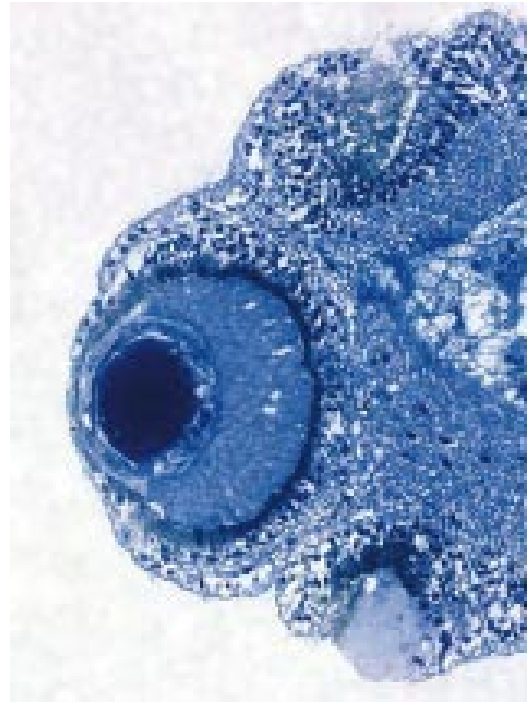

Histopathological photograph of smaller of two "camera" style eyes. Pathology performed by Thomas $\mathrm{N}$ Blankenship, PhD.

near the lower edge of the bell. Each club-shaped rhopalium is surrounded with petal-like leaves and represent the sensory elements of the beast. There are six eyes on each rhopalium. Four of these eyes, called ocelli, are simple, and probably can only perceive light and dark. Two of these eyes, however, are surprisingly complex.

Each of the eight complex eyes (two per rhopalium) has a cornea, lens, and a multilayered retina. The cornea consists of a flattened layer of epithelium directly in contact with the lens without an intervening chamber or iris. The spherical lens is well organised having a novel family of crystalline polypeptides with a decreasing index of refraction (Piatigorsky JJ, et al, J Comp Phys A Sensory Neural Behav Physiol 1989;164:577-88) as seen in other aquatic species discussed briefly in the May 2002 BJO cover essay. The lens allows for improved focusing and minimises spherical aberration. The retina, or at least the tissue immediately behind the lens, consists of a vitreous layer, ciliated photoreceptors with pigment granules, and a nuclear layer with neurons extending to a circular neural ring connecting the other rhopalia. The photoreceptors are described as everted since the distal end of the cells containing the visual pigment are directly behind the lens without the intervening neural tissue as would be seen in vertebrates. The cubozoan species that have been investigated direct the larger of the two complex eyes towards the interior of the bell, and smaller one to view outwards, and/or upwards towards the apex of the bell. The organisms are phototactic, to be sure, but these photoreceptors contain rhodopsin and other visual pigments including opsins for blue, green and ultraviolet light (Martin VJ, Can J Zool 2002;80:1703). Although the retina probably cannot fully exploit the focusing ability of the lens, the lens is essential for the spatial resolution and sensitivity that are present in the two eyes with lenses. $T$ cystophora's eyes are sensitive to a dynamic range of intensity in visible light and, apparently, colour (Coates MM, et al, Am Zoo 2000;40:977). There is evidence that the eyes are able to resolve images and perceive motion, at least to some extent. An eye with this visual sophistication is not seen evolutionarily again for millions of years.

Although the complex "camera" style eyes are surprisingly well organised, image processing is another matter. Although there is no central processing, the first order neurons progressing from the photoreceptors do connect to the decentralised neural ring which connects the rhopalia. There are small "sensory mini-ganglia" that probably perform some form of neural processing and coordination. There must be some form of communication between the eyes, the bell, and the tentacles because these species are predatorial, and coordination is necessary. The multiple eye types are probably specialised to collect different visual information leading to a sort of "hard wiring" of the visual system and reduction of input processing.

Some cubozoans actually may "chase" fish with tentacles; $C$ fleckeri of the north eastern Australian waters can swim at up to 3-4 knots per hour. Although most jellyfish, including $T$ cystophora, feed on plankton, some of the larger jellyfish actually kill fish by using their potent venom; presumably the venom is so potent so that the jellyfish can kill quickly and avoid harm to itself.

The sensory inputs from the rhopalia are integrated and communicated to the bell and tentacles without a central processing centre. So, maybe, the eye doesn't need a brain, and perhaps the brain is an outpouching of the eye.

I R Schwab, M M Coates irschwab@ucdavis.edu Specimen courtesy of Joseph Horwitz, PhD, and Joram Piatigorsky, PhD. 